\title{
Synthesis of Colloidal 2D/3D MoS 2 Nanostructures by Pulsed Laser Ablation in an Organic Liquid Environment
}

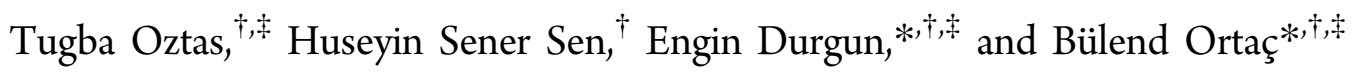

${ }^{\dagger}$ UNAM-National Nanotechnology Research Center, and ${ }^{\ddagger}$ Institute of Materials Science and Nanotechnology, Bilkent University, Ankara 06800, Turkey

ABSTRACT: Two-dimensional $\mathrm{MoS}_{2}$ nanosheets $\left(2 \mathrm{D} \mathrm{MoS}\right.$ NS) and fullerene-like $\mathrm{MoS}_{2}$ nanostructures (3D $\mathrm{MoS}_{2} \mathrm{NS}$ ) with varying sizes are synthesized by nanosecond laser ablation of hexagonal crystalline $2 \mathrm{H}-\mathrm{MoS}_{2}$ powder in organic solution (methanol). Structural, chemical, and optical properties of $\mathrm{MoS}_{2}$ NS are characterized by optical microscopy, scanning electron microscopy, transmission electron microscopy, X-ray diffraction, and Raman and UV-vis-near infrared absorption spectroscopy techniques. Results of the structural analysis show that the obtained $\mathrm{MoS}_{2} \mathrm{NS}$ mainly present a layered morphology from micrometer to nanometer sized surface area. Detailed analysis of the product also proves the existence of inorganic polyhedral fullerene-like 3D $\mathrm{MoS}_{2} \mathrm{NS}$ generated by pulsed laser ablation in methanol. The possible factors which may lead to formation of both 2D and 3D $\mathrm{MoS}_{2}$ NS in methanol are examined by ab initio calculations and shown to correlate with vacancy formation. The hexagonal crystalline structure of $\mathrm{MoS}_{2} \mathrm{NS}$ was determined by XRD analysis. In Raman spectroscopy, the peaks at 380.33

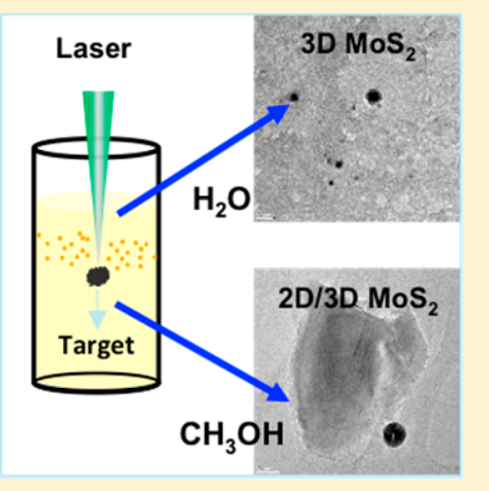
and $405.79 \mathrm{~cm}^{-1}$ corresponding to the $\mathrm{E}_{2 \mathrm{~g}}^{1}$ and $\mathrm{A}_{1 \mathrm{~g}}$ phonon modes of $\mathrm{MoS}_{2}$ were clearly observed. The colloidal MoS $\mathrm{NS}_{2}$ solution presents broadband absorption edge tailoring from the UV region to the NIR region. Investigations of $\mathrm{MoS}_{2} \mathrm{NS}$ show that the one-step physical process of pulsed laser ablation-bulk $\mathrm{MoS}_{2}$ powder interaction in organic solution opens doors to the formation of "two scaled" micrometer- and nanometer-sized layered and fullerene-like morphology $\mathrm{MoS}_{2}$ structures.

\section{INTRODUCTION}

The synthesis of semiconductor nanomaterials attracts a great deal of interest because of the physical, chemical, electrical, and optical properties of the nanomaterials. ${ }^{1}$ The size- and shapedependent properties of semiconductor nanomaterials possess potential applications in new nanomaterials-based photonics and optoelectronics devices. ${ }^{2}$ One of the most rapidly growing scientific areas is the generation technique of nanocrystals from group IV elements. Nanocrystals from this group are twodimensional (2D) honeycomb lattice (such as graphene) semiconductor materials. Alternately, $\mathrm{MoS}_{2}$ is a newly emerging transition-metal dichalcogenide semiconductor material. Because of its natural bandgap $(\sim 1.2 \mathrm{eV}$ indirect bandgap in multilayer/bulk form, $\sim 1.85 \mathrm{eV}$ direct bandgap in monolayer form), $\mathrm{MoS}_{2}$ presents advantageous properties compared to group IV semiconductor or graphene in many applications. ${ }^{3,4}$ It is also shown that both microscaled $\mathrm{MoS}_{2}$ and nanoscaled $\mathrm{MoS}_{2}$ have perfect resistance against oxidation in a moist air environment, which makes them more durable in device fabrication compared to group IV semiconductor nanosheets (NS). ${ }^{5,6}$

In literature, the existence of $\mathrm{MoS}_{2} \mathrm{NS}$ in the form of fullerene-like NS have been predicted and experimentally demonstrated. ${ }^{7}$ The importance of such a kind of structures is due to the presence of peculiar features. It is now recognized that polyhedral closed-caged NS under certain energetic considerations are thermodynamically more stable than isolated basal sheets of the lamellar structure. ${ }^{8}$ These $\mathrm{MoS}_{2}$ NS have attracted considerable attention recently because of their potential use in microlubrication, ${ }^{9}$ oil refinement, ${ }^{10}$ photocatalysis, $^{11}$ and photodetector applications. ${ }^{12}$

On the other hand, $\mathrm{MoS}_{2}$ has interesting properties in the case of a $2 \mathrm{D}$ ultrathin atomic layer structure. The unique properties of $2 \mathrm{D} \mathrm{MoS} 2 \mathrm{NS}$ make them a perfect alternative material for heterogeneous catalysis, hydrogen storage, lithium-magnesium ion batteries, ${ }^{13,14}$ and various biomedical applications. ${ }^{13}$ Since $\mathrm{MoS}_{2}$ NS possess promising photoelectric properties that are tunable by physical layer thickness of $2 \mathrm{D}$ $\mathrm{MoS}_{2}$ NS, various electronic and optoelectronic devices are fabricated based on $\mathrm{MoS}_{2} \cdot{ }^{15}$ Despite the fact that single-layer $\mathrm{MoS}_{2}$ has a large direct band gap of $1.8 \mathrm{eV}$ and low electron mobility, a single-layer transistor based on $\mathrm{MoS}_{2}$ has been developed. ${ }^{16}$ This work has been a great indicator to the usage of $\mathrm{MoS}_{2}$ in optoelectronic applications. For $\mathrm{MoS}_{2}$-based transistor applications, it is even possible to achieve an applicable electron mobility level. In the literature, it was also shown that $\mathrm{MoS}_{2}$ is a potential candidate in solar cell applications. ${ }^{17,18}$ Various properties and possible applications of $2 \mathrm{D}-\mathrm{MoS}_{2}$ and its nanoribbons have also been an active subject of theoretical studies. ${ }^{19-23}$ All these recent research results clearly demonstrate that $2 \mathrm{D} \mathrm{MoS}_{2} \mathrm{NS}$ present a great potential for nanoelectronic and nanophotonic applications.

There are various methods to synthesize $2 \mathrm{D}$ and $3 \mathrm{D} \mathrm{MoS}_{2}$ NS including mechanical exfoliation, ${ }^{24,25}$ solution-based ex-

Received: June 12, 2014

Revised: September 28, 2014

Published: November 24, 2014 
foliation, ${ }^{26}$ CVD-based synthesis, ${ }^{27}$ thermal decomposition, ${ }^{28}$ powder sublimation, ${ }^{29}$ and electrochemical/chemical synthesis. ${ }^{5}$ In addition, most of the synthesis approaches for the formation of $\mathrm{MoS}_{2}$ NS require hazardous compounds such as $\mathrm{H}_{2} \mathrm{~S}$ and $\mathrm{H}_{2}$ with difficulties in handling and storage. ${ }^{30}$ Pulsed laser ablation (PLA) is another promising method to generate $2 \mathrm{D}$ and $3 \mathrm{D} \mathrm{NS} .{ }^{13}$ The usage of unique scientific facilities of laser-matter interaction allows the generation of a wide variety of noble $\mathrm{NS}^{31}$ and semiconductor nanocrystals ${ }^{32}$ with different structural morphologies. Compared to other methods; PLA, especially in liquids, is a versatile method of generating colloidal, highly pure and agent-free NS. In the case of $\mathrm{MoS}_{2}$ NS generation with PLA, chemical precursors are definitely not required. There have been a few reports about fullerene-like 3D $\mathrm{MoS}_{2}$ NS generation by laser ablation technique only in water. $\mathrm{Wu}$ et al. showed that $3 \mathrm{D} \mathrm{MoS}_{2} \mathrm{NS}$ obtained through laser ablation are fullerene-like and have good solubility and also are also biocompatible in nature, which makes 3D $\mathrm{MoS}_{2} \mathrm{NS}$ applicable in various biomedical areas. ${ }^{13}$

Here, we present a relatively simple, one step, faster method for the synthesis of different $\mathrm{MoS}_{2} \mathrm{NS}$ morphologies in organic liquid. The use of the PLA technique to crystalline $2 \mathrm{H}-\mathrm{MoS}_{2}$ powder in methanol generates both colloidal 2D and 3D $\mathrm{MoS}_{2}$ NS. The majority of $\mathrm{MoS}_{2}$ NS produced by PLA have a layered morphology from large size (micrometer) to small size (nanometer). Other parts of our sample consist of inorganic polyhedral fullerene-like 3D $\mathrm{MoS}_{2}$ NS. In addition, ab initio calculations are performed in order to reveal the possible factors which may lead to different morphologies. The optical microscope analysis, scanning electron microscopy (SEM), transmission electron microscopy (TEM), X-ray diffraction (XRD), Raman and UV-vis-near infrared (NIR) absorption measurements were used to further understand structure, composition, size, chemical, and optical properties of $\mathrm{MoS}_{2} \mathrm{NS}$. XRD analysis provided evidence about formation of the hexagonal crystalline structure $\mathrm{MoS}_{2}$ NS. In Raman spectroscopy, generation of the crystalline nature of the $\mathrm{MoS}_{2}$ was confirmed. The colloidal $\mathrm{MoS}_{2}$ NS solution presented broadband absorption edge tailoring from the NIR region to the UV region.

\section{EXPERIMENTAL SECTION}

Bulk $\mathrm{MoS}_{2}$ powder (99.99\%, Sigma-Aldrich) and pure methanol ( $>99 \%$, Sigma-Aldrich) were used as-received without any additional purification. Colloidal $\mathrm{MoS}_{2} \mathrm{NS}$ solution was generated by using the PLA technique in methanol. The commercial nanosecond pulsed ND:YLF laser operated at 527 $\mathrm{nm}$ with a pulse duration of $100 \mathrm{~ns}$, average output power of 16 $\mathrm{W}$ at a pulse repetition rate of $1 \mathrm{kHz}$ corresponding to a pulse energy of $16 \mathrm{~mJ}$ being used. A $1 \mathrm{mg}$ portion of bulk $\mathrm{MoS}_{2}$ powder was added to $10 \mathrm{~mL}$ of pure methanol for the PLA experiment. The laser beam was focused on the bulk $\mathrm{MoS}_{2}$ powder target, which is placed in a glass vial containing $10 \mathrm{~mL}$ of methanol, using a plano-convex lens with a focal length of 50 $\mathrm{mm}$. The PLA process was carried out for $15 \mathrm{~min}$. To obtain a well dispersed NS solution, the colloidal NS were continuously stirred by a magnetic stirrer at $800 \mathrm{rpm}$ during the laser ablation process. The color of the final product became dark-orange. Then, the produced NS were characterized via optical microscope analysis, SEM, TEM, XRD, Raman spectroscopy, and UV-vis spectroscopy to get information in detail about their physical and chemical properties.
To investigate the atomistic nature, structural effects, and $\mathrm{MoS}_{2}$-solvent interaction, we used first-principles computational techniques based on density functional theory, ${ }^{33,34}$ implemented in the Vienna ab initio simulation package. ${ }^{35,36}$ The exchange-correlation potential was approximated within the generalized gradient approximation (GGA) using PBE functional ${ }^{37}$ including van der Waals correction $(\mathrm{VdW})^{38}$ and projector augmented-wave $(\mathrm{PAW})^{39}$ potentials. The calculations for nanomeshes were done at $\Gamma$-point using a plane-wave basis set with a kinetic energy cutoff of $500 \mathrm{eV}$. All structures were optimized with simultaneous minimization of the total energy and interatomic forces. The convergence on the total energy and force was set to $10^{-5} \mathrm{eV}$ and $10^{-2} \mathrm{eV} / \AA$, respectively.

\section{RESULTS AND DISCUSSION}

The first structural investigation of $\mathrm{MoS}_{2} \mathrm{NS}$ was performed by using an optical microscope (Carl Zeiss, Axio Imager). Figure 1
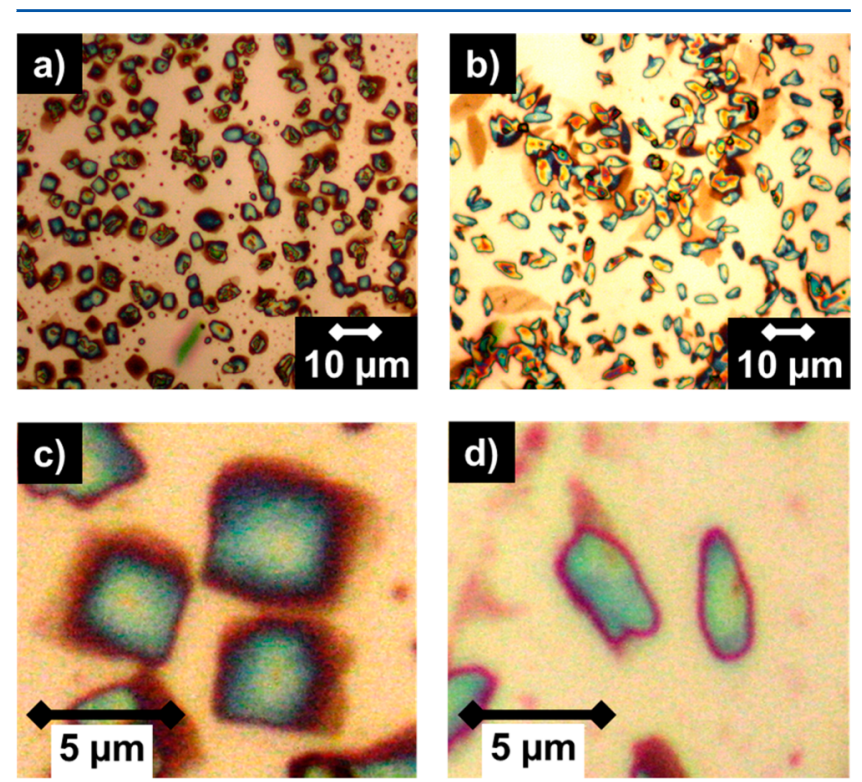

Figure 1. Optical microscope images of $\mathrm{MoS}_{2} \mathrm{NS}$ showing micrometer size layered morphology on a silicon substrate.

shows the optical microscope images of $\mathrm{MoS}_{2}$ NS. The images clearly prove that the obtained $\mathrm{MoS}_{2}$ NS have layered morphology, and their sizes reach up to the micrometer scale. We also observed that $2 \mathrm{D} \mathrm{MoS}_{2}$ NS have a quadratic shape or elliptical-like structure.

The morphology of $\mathrm{MoS}_{2}$ NS was then studied by using SEM (FEI, Quanta 200 FEG) at an accelerating voltage to 20 $\mathrm{kV}$ to be able to get information in detail about their structure. SEM images (see Figure 2) demonstrate that 2D $\mathrm{MoS}_{2} \mathrm{NS}$ were produced on both the microscale and nanoscale. On the other hand, we recognize that $3 \mathrm{D} \mathrm{MoS}$ NS were also produced by using a one-step PLA technique in methanol. It is understood that the majority of $\mathrm{MoS}_{2}$ NS have a layered shape and the size of the $3 \mathrm{D} \mathrm{MoS}_{2} \mathrm{NS}$ is relatively small.

The morphology and the elemental analysis of the $\mathrm{MoS}_{2} \mathrm{NS}$ drop-cast onto carbon-coated TEM grid were also performed by using FEI-Tecnai $\mathrm{G}^{2} \mathrm{~F} 30$ at an operating voltage of about $300 \mathrm{kV}$ equipped with an energy dispersive X-ray spectroscopy (EDS) system. Figure 3 indicates that the morphology of the final 2D $\mathrm{MoS}_{2}$ NS product presents both few and multilayer 

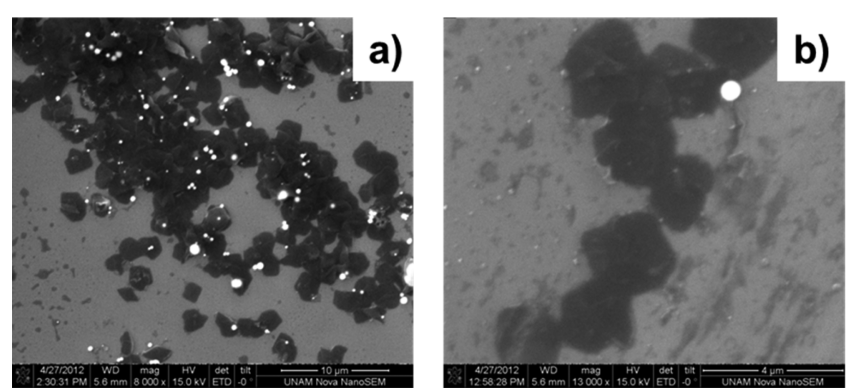

Figure 2. Representative SEM images of $\mathrm{MoS}_{2}$ NS obtained by PLA in an organic liquid. SEM images confirm 2D MoS2 NS and 3D MoS2 NS production in one step.
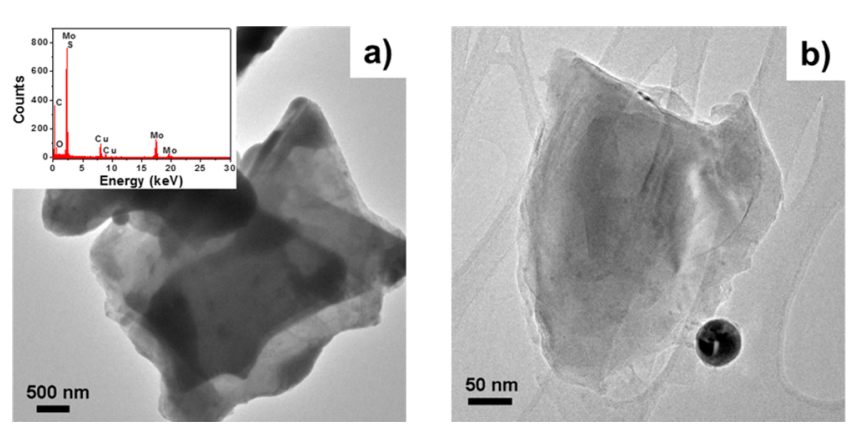

Figure 3. Representative TEM images of the $\mathrm{MoS}_{2}$ NS indicating multilayer and few NS by the PLA of bulk $\mathrm{MoS}_{2}$ powder in methanol. Inset, EDS spectrum.

NS, and their surface area sizes vary from the micrometer to nanometer scale. Furthermore, EDS analysis shows that our sample includes only $\mathrm{Mo}, \mathrm{S}, \mathrm{C}, \mathrm{Cu}$, and $\mathrm{O}$ atoms. The peaks related to carbon $(\mathrm{C})$, oxygen $(\mathrm{O})$, and copper $(\mathrm{Cu})$ are associated with the TEM grid used. The presence of the Mo and $S$ peaks in the EDS spectrum confirms that $\mathrm{MoS}_{2}$ NS were successfully generated by the PLA technique with a small of amount of impurities observed.

TEM results in detail are given in Figure 4. This figure shows that $3 \mathrm{D} \mathrm{MoS}_{2}$ NS have a fullerene-like crystalline structure, and
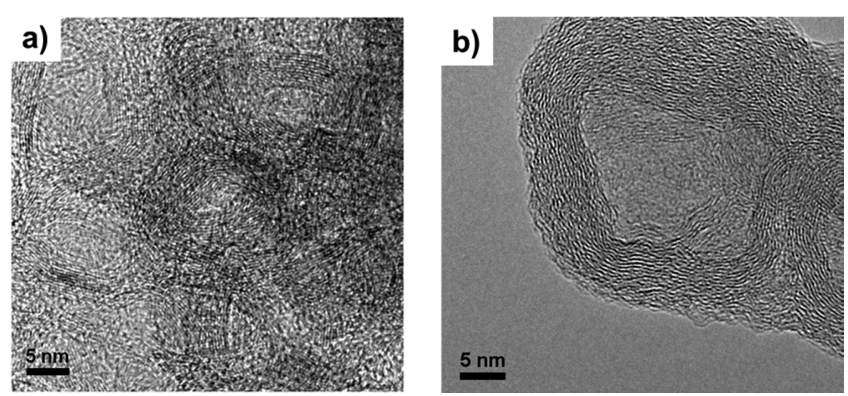

Figure 4. HRTEM images of $3 \mathrm{D} \mathrm{MoS}_{2} \mathrm{NS}$ and zoom of single isolated 3D $\mathrm{MoS}_{2}$ nanosheet showing fullerene-like structure.

it also clearly demonstrates that the diameters of the generated 3D $\mathrm{MoS}_{2}$ NS are in the range of few nanometers.

In our previous study, we produced $\mathrm{MoS}_{2}$ NS with the PLA technique in DI water and we only observed 3D $\mathrm{MoS}_{2}$ NS generation. $^{12}$ In this study, we repeat the same procedure except for the solvent type, which is methanol instead of water; we recognize that the obtained NS have different morphologies. The use of the PLA technique to crystalline $2 \mathrm{H}-\mathrm{MoS}_{2}$ powder in methanol generates both colloidal $2 \mathrm{D}$ and $3 \mathrm{D} \mathrm{MoS}_{2}$ NS. In this respect, we analyze the factors, which may drastically affect the nanoparticles structure, by ab initio methods.

In literature it has been proposed that nanoparticles of layered compounds can be unstable against folding and close into fullerene-like structures. ${ }^{40}$ The folding and rolling of $2 \mathrm{D}$ nanosheets have been experimentally observed. ${ }^{41,42}$ Moreover a scroll-like morphology of the folded structures is considered to be a possible route to inorganic fullerenes. ${ }^{43}$ Following these discussions, in our model, we start with ideal (without any defects) monolayer $\mathrm{MoS}_{2}$ nanomeshes with varying sizes (Figure 5) and consider the deviation from planar structure

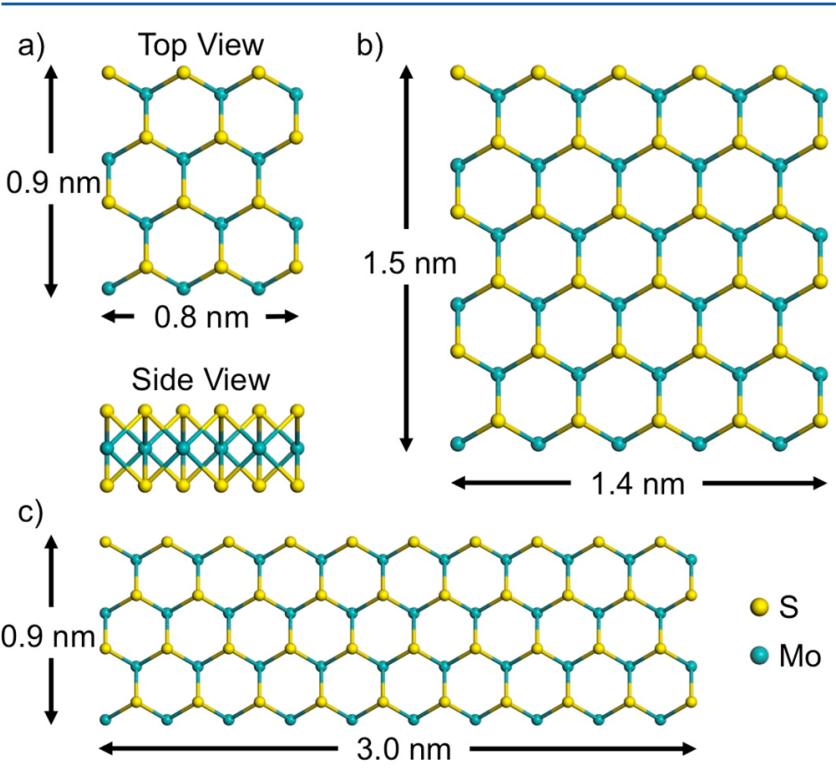

Figure 5. Side and top view of $\mathrm{MoS}_{2}$ nanomeshes with varying sizes. Blue and yellow spheres indicate Mo and $S$ atoms, respectively.

as an indication of a clustering tendency. In our experimental results, the typical size for nanosheets is larger than $50 \mathrm{~nm}$, whereas it is around $5 \mathrm{~nm}$ for nanoparticles, which indicates that the clustering occurs for relatively small sizes and there is no need to analyze particle sizes larger than $5 \mathrm{~nm}$. In this respect we only cover the size range between 1 and $3 \mathrm{~nm}$. When we optimize the structures in vacuum by minimizing the total energy and forces on the atoms, all ideal nanomeshes are found to be planar except for small distortions at the edges (which are expected due to the broken bonds). Even when we break the symmetry of the system and deform the planar structures as shown in Figure 6, the nanomeshes revert back to a)

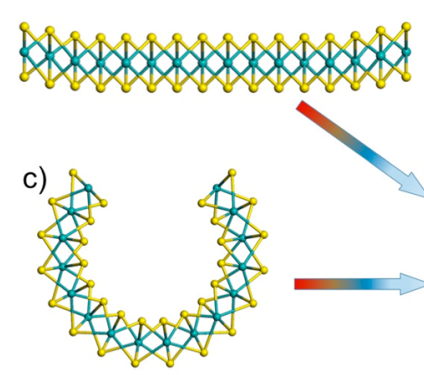

b)

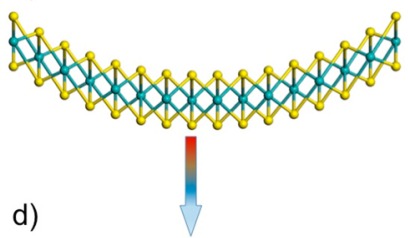

d)

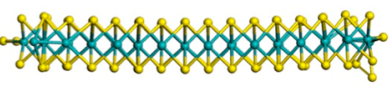

Figure 6. $(a-c)$ initial (deformed) and (d) final (optimized) structures of $\mathrm{MoS}_{2}$ nanomesh. 
their ideal planar form after geometry optimization. When compared, the planar form is even energetically more favorable $(\sim 0.2 \mathrm{eV} /$ atom $)$ than the completely curved system in which all dangling bonds are eliminated. ${ }^{44}$

To investigate the effect of solvents, we first examine the interaction of water and methanol molecules with $\mathrm{MoS}_{2}$ nanomeshes starting from a single molecule adsorption. Both molecules do not bind to the $\mathrm{MoS}_{2}$ nanomesh surface ${ }^{45,46}$ as shown in Figure 7. The adsorption energy $\left(E_{\mathrm{a}}\right)$ is calculated to

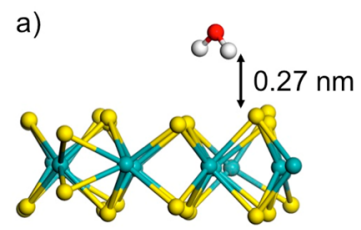

b)
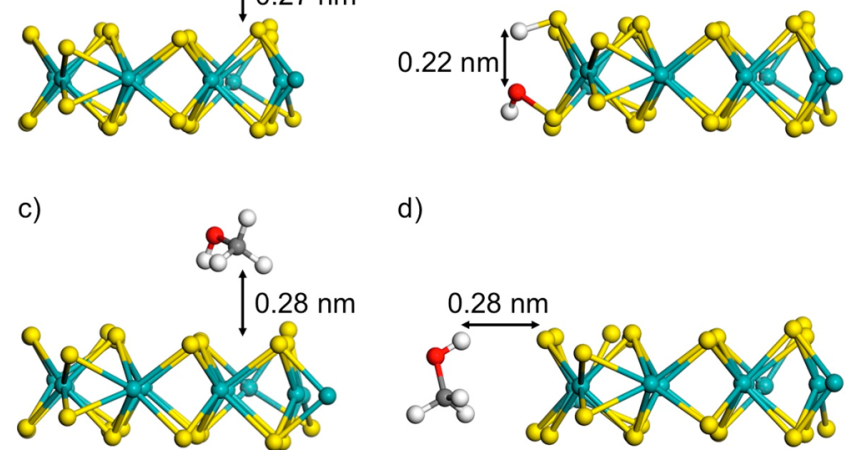

d)

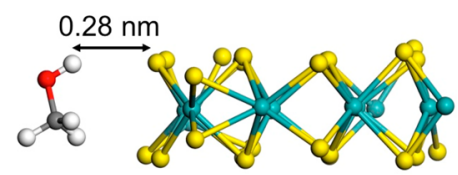

Figure 7. Single $\mathrm{H}_{2} \mathrm{O}$ molecule (a) adsorption on surface (b) and dissociation at the edge; and single $\mathrm{CH}_{3} \mathrm{OH}$ molecule adsorption (c) on surface (d) at the edge of $\mathrm{MoS}_{2}$ nanomesh.

be 0.3 and $0.4 \mathrm{eV} /$ molecule for water and methanol, respectively. When VdW interactions are included, $E_{\mathrm{a}} \approx 0.1$ $\mathrm{eV}$ increases for both molecules. Alternately, a single $\mathrm{H}_{2} \mathrm{O}$ strongly interacts with the edges and even can dissociate, ${ }^{47}$ while $\mathrm{CH}_{3} \mathrm{OH}$ remains intact. Next, we gradually increase the number of surrounding molecules to resemble the solvent medium. In a similar manner, except for $\mathrm{H}_{2} \mathrm{O}$ molecules at the edges, neither methanol nor water molecules interact with $\mathrm{MoS}_{2}$ nanomesh and do not modify the planar geometry even when the VdW interactions are taken into account. We obtain similar results when we increase the temperature, once again indicating a tendency for nanosheet formation instead of clustering.

Our analysis shows that $\mathrm{MoS}_{2} \mathrm{NS}$ are successfully generated by the PLA technique, with no chemical impurities but intrinsic structural defects in monolayer $\mathrm{MoS}_{2} \mathrm{NS}$ can occur during the formation process. Accordingly we consider possible defects, namely, S, Mo, Mo-S, Mo-2S vacancies in nanomeshes. The geometry optimization results at different temperatures demonstrate that the planar structures are deformed upon introducing defects. The amount of deformation is stronger for the S-vacancy case (Figure 8a) but also significant for other types as well. Interestingly, while the presence of $\mathrm{H}_{2} \mathrm{O}$ molecules around a nanomesh enhances deformation (Figure $8 \mathrm{~b}), \mathrm{CH}_{3} \mathrm{OH}$ molecules reduce the effect (Figure $8 \mathrm{c}$ ).

Finally, depending on our first-principles calculations, we propose that the production of nanoclusters by the PLA method is linked with the amount of vacancy formation, as ideal structures have a tendency to stay in the planar form. While the interaction between the water molecules and nanomesh enhances the deformation resulting from defects, surrounding methanol molecules reduce the amount of deformation. Thus, using methanol as solvent increases the possibility of nanosheet formation which can explain the a) $\mathrm{MoS}_{2}$ with $\mathrm{S}$ defect

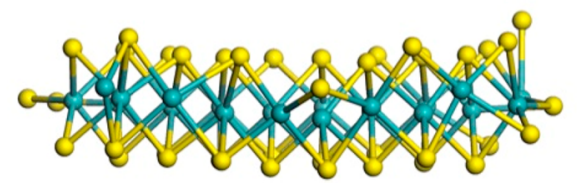

b) Addition of $\mathrm{H}_{2} \mathrm{O}$

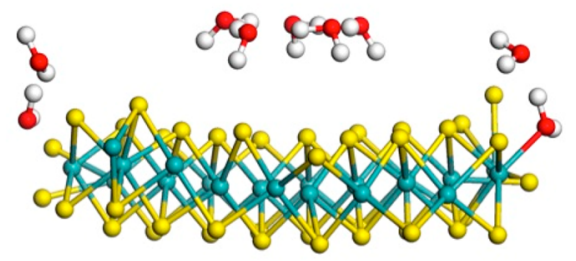

c) Addition of $\mathrm{CH}_{3} \mathrm{OH}$

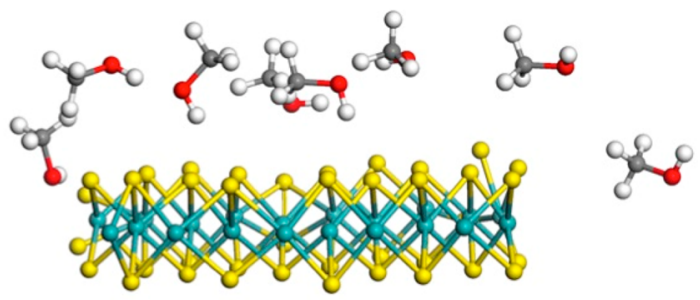

Figure 8. Optimized structures of $\mathrm{MoS}_{2}$ nanomesh with a single sulfur defect (a) in vacuum, (b) with surrounding $\mathrm{H}_{2} \mathrm{O}$ molecules, and (c) with surrounding $\mathrm{CH}_{3} \mathrm{OH}$ molecules.

formation of both colloidal 2D and 3D $\mathrm{MoS}_{2} \mathrm{NS}$ in methanol distinct from water.

To better understand the crystallographic structure of $\mathrm{MoS}_{2}$ NS, an XRD study was performed. The PANalytical X'Pert PRO multipurpose diffractometer operated at a voltage about $45 \mathrm{kV}$ and a current of $40 \mathrm{~mA}$ using a $\mathrm{CuK} \alpha$ radiation source was used. The $\mathrm{MoS}_{2}$ NS sample was prepared by depositing and drop-casting the $\mathrm{MoS}_{2}$ NS on a low-intensity background silicon (100) substrate. XRD measurement analysis was carried out to determine the crystalline structure and the composition of the $\mathrm{MoS}_{2}$ NS. First we studied XRD analysis of $2 \mathrm{H}-\mathrm{MoS}_{2}$ powder. Nine sharp diffraction peaks at $14.43^{\circ}, 29^{\circ}, 32.7^{\circ}$, $33.46^{\circ}, 35.85^{\circ}, 38.56^{\circ}, 44.15^{\circ}, 49.82^{\circ}$, and $58.35^{\circ}$ corresponding to the (002), (004), (100), (101), (102), (103), (006), (105), and (110) reflections of hexagonal $2 \mathrm{H}-\mathrm{MoS}_{2}$ with lattice constants $a=3.160, c=12.295 \AA$ (ICDD-JPDS card No. 391492) are observed. ${ }^{13}$ No other diffraction peaks were observed indicating that the $2 \mathrm{H}-\mathrm{MoS}_{2}$ powder used for this experiment was of a crystalline structure. The XRD pattern of $\mathrm{MoS}_{2} \mathrm{NS}$ is shown in Figure 9. Five main sharp diffraction peaks at 14.46 (002), 29.08 (100), 39.61 (103), 49.84 (105), and 60.2 (110) are clearly observed. ${ }^{13}$ This result indicates that $\mathrm{MoS}_{2} \mathrm{NS}$ with a crystalline structure were successfully generated by the PLA technique.

Raman spectroscopy is a very useful technique for the structural characterization of nanomaterials. Raman spectroscopy of $\mathrm{MoS}_{2}$ NS was performed using a Witec Alpha 300S Micro Raman spectrometer with a Nd:YAG laser at an excitation wavelength $532 \mathrm{~nm}$ (laser power, $10 \mathrm{~mW}$ ) and Nikon $100 \times($ N.A. $=0.9$ ) air objective, and the Raman spectrum was recorded at room temperature. The sample was 


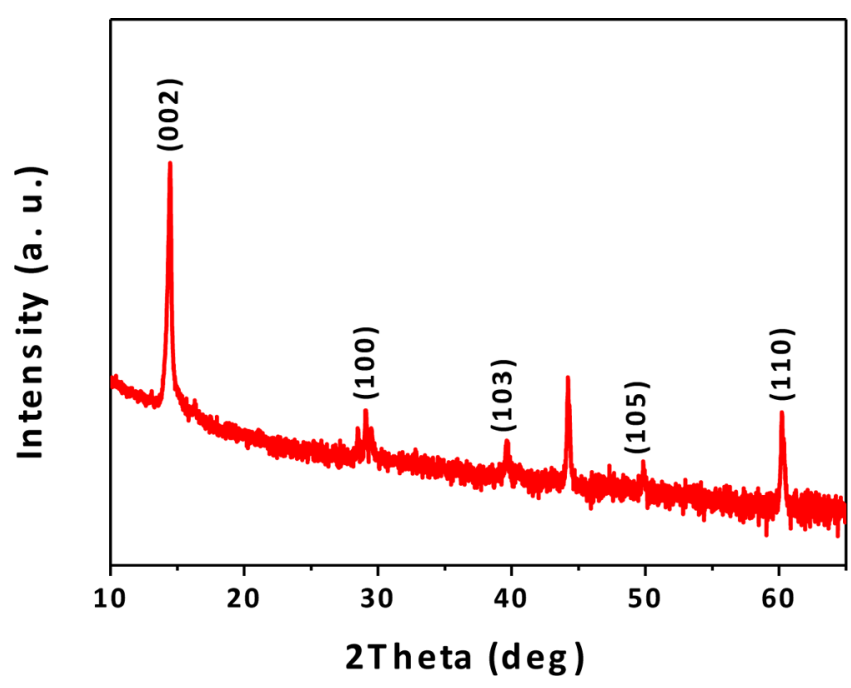

Figure 9. XRD pattern of $\mathrm{MoS}_{2}$ NS drop-cast onto a low intensity background silicon (100) substrate showing reflections characteristic of the crystalline structure of $\mathrm{MoS}_{2}$.

obtained by drop-casting the $\mathrm{MoS}_{2}$ NS onto a silicon wafer for optical microscopy, SEM, and Raman analysis. Raman spectroscopy is a widely applicable technique to investigate optical and structural properties of nanomaterials. Further evaluations can be made for vibrational modes of $\mathrm{MoS}_{2} \mathrm{NS}$ in Raman spectroscopy. The Raman spectrum of $\mathrm{MoS}_{2} \mathrm{NS}$, which is given in Figure 10, shows two peaks centered at 381.7 and 407.5

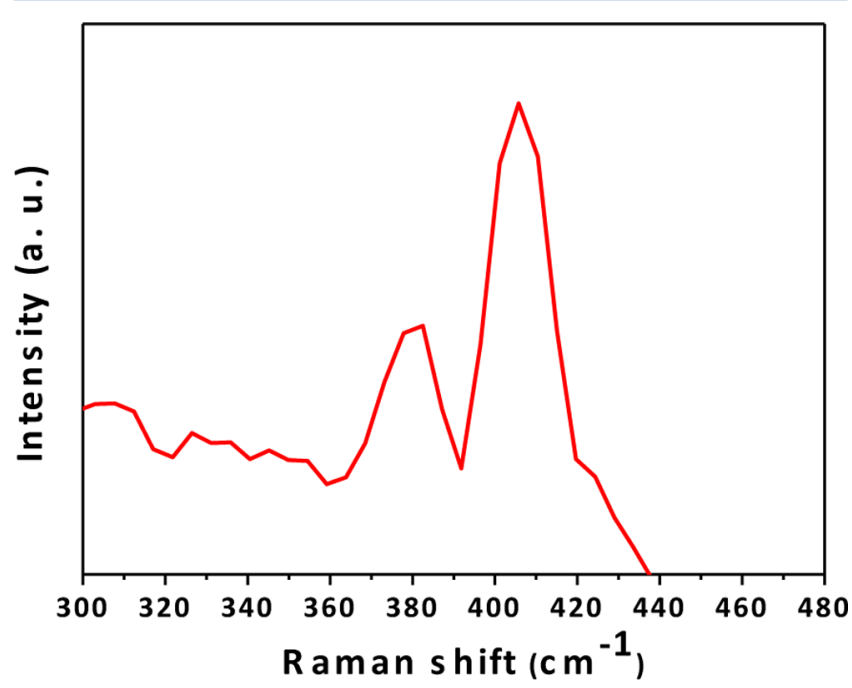

Figure 10. Raman spectra of $\mathrm{MoS}_{2}$ NS generated by nanosecond laser ablation in methanol.

$\mathrm{cm}^{-1}$. In literature, these peaks are attributed to in-plane $\mathrm{E}_{2 \mathrm{~g}}^{1}$ and out-of-plane $\mathrm{A}_{1 \mathrm{~g}}$ vibrations of $\mathrm{MoS}_{2}$, respectively. ${ }^{13}$ The Raman result also provides that the obtained nanomaterials are $\mathrm{MoS}_{2}$ NS.

The optical absorption spectrum of $\mathrm{MoS}_{2}$ NS was obtained with a Varian Cary 5000 UV-vis-NIR spectrophotometer operating in the 200-1300 nm wavelength range. The $\mathrm{MoS}_{2}$ NS solution was added into a quartz cuvette for optical absorption measurement. $\mathrm{MoS}_{2}$ is a new semiconductor material exhibiting structure-dependent optical properties. Figure 11 shows normalized optical absorption spectra of $\mathrm{MoS}_{2}$ NS in methanol. The optical absorption spectrum of

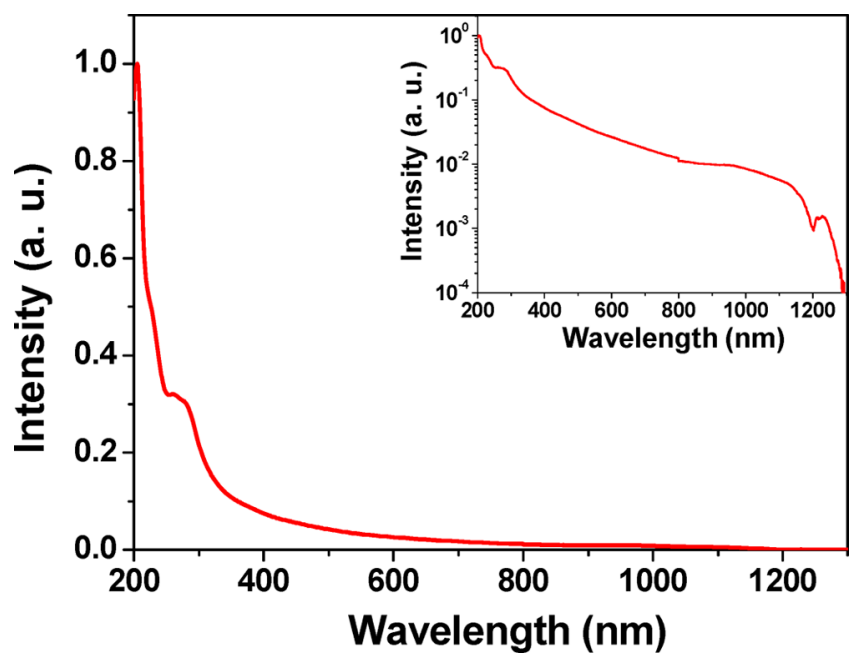

Figure 11. UV-vis absorption spectrum of $\mathrm{MoS}_{2}$ NS in methanol showing broadband optical absorption behavior.

colloidal $\mathrm{MoS}_{2}$ NS shows a minimum optical absorption feature at $1300 \mathrm{~nm}$ and also prominent shoulders at $265 \mathrm{~nm}$, and the strong rising absorption edge shifts toward the UV region. Compared to the optical properties of $3 \mathrm{D} \mathrm{MoS}_{2} \mathrm{NS}$ from the PLA technique in DI water, ${ }^{12}$ laser-generated $\mathrm{MoS}_{2}$ NS have broadband optical absorption properties tailoring from the NIR region to the UV region, and therefore, $\mathrm{MoS}_{2} \mathrm{NS}$ can be considered as a prime candidate for various photonics and optoelectronics applications.

\section{CONCLUSION}

Different-shaped nanostructures of $\mathrm{MoS}_{2}$ have been synthesized through a one-step PLA technique of hexagonal crystalline $2 \mathrm{H}-\mathrm{MoS}_{2}$ powder in organic liquid. Structural analysis of colloidal nanocomposites demonstrated that the obtained $\mathrm{MoS}_{2}$ product presents layered morphology with micrometer- to nanometer-sized surface area structures. The inorganic fullerene-like $\mathrm{MoS}_{2} \mathrm{NP}$ are also successfully synthesized in a one-step technique. $\mathrm{Ab}$ initio calculations indicate that the formation of fullerene-like structures is linked with vacancies. Methanol reduces the deformation resulting from vacancies, which can clarify the concurrent production of nanoclusters and nanosheets. The synthesized $\mathrm{MoS}_{2} \mathrm{NS}$ gave characteristic Raman peaks at 381.7 and $407.5 \mathrm{~cm}^{-1}$ that are attributed to the in-plane $E_{2 g}^{1}$ and out-of-plane $A_{1 g}$ vibration modes of $\mathrm{MoS}_{2}$ and $\mathrm{MoS}_{2}$ NS having a hexagonal crystal structure. Broadband optical absorption behavior was observed for the colloidal $\mathrm{MoS}_{2}$ NS synthesized in organic solution by pulsed laser ablation. Our study revals a one-step synthesis method for the generation of "two scales-(2D and 3D)" micrometer- and nanometer-sized layered and spherical fullerene-like morphology $\mathrm{MoS}_{2}$ structures.

\section{AUTHOR INFORMATION}

\section{Corresponding Authors}

*E-mail: ortac@unam.bilkent.edu.tr.

*E-mail: durgun@unam.bilkent.edu.tr.

Notes

The authors declare no competing financial interest. 


\section{ACKNOWLEDGMENTS}

The State Planning Organization (DPT) of Turkey is acknowledged for the support of UNAM-Institute of Materials Science and Nanotechnology. This work was partially supported by TUBITAK under Project No. 113T050. Dr. Ortaç acknowledges the 'Industrial Thesis Projects Programme' of the Ministry of Industry and Trade for funding the San-Tez (636.STZ.2010-1) project. E.D. acknowledges support from Bilim Akademisi, The Science Academy, Turkey under the BAGEP program. Ab initio calculations were performed at TUBITAK ULAKBIM, High Performance and Grid Computing Center (TR-Grid e-Infrastructure). The authors thank to $\mathrm{H}$. A. Vural for his assistance in the Raman experiments.

\section{REFERENCES}

(1) Trindade, T.; O’Brien, P.; Pickett, N. L. Nanocrystalline Semiconductors: Synthesis, Properties, and Perspectives. Chem. Mater. 2001, 13, 3843-3858.

(2) Lauritsen, J. V.; Kibsgaard, J.; Helveg, S.; Topsøe, H.; Clausen, B. S.; Lægsgaard, E.; Besenbacher, F. Size-Dependent Structure of $\mathrm{MoS}_{2}$ Nanocrystals. Nat. Nanotechnol. 2007, 2, 53-58.

(3) Qiu, H.; Pan, L.; Yao, Z.; Li, J.; Shi, Y.; Wang, X. Electrical Characterization of Back-Gated Bi-Layer $\mathrm{MoS}_{2}$ Field-Effect Transistors and the Effect of Ambient on Their Performances. Appl. Phys. Lett. 2012, 100, 123104-123104-3.

(4) Yu, Y.; Li, C.; Liu, Y.; Su, L.; Zhang, Y.; Cao, L., Controlled Scalable Synthesis of Uniform, High-Quality Monolayer and FewLayer $\mathrm{MoS}_{2}$ Films. Sci. Rep. 2013, 3.

(5) Li, Q.; Newberg, J.; Walter, E.; Hemminger, J.; Penner, R. Polycrystalline Molybdenum Disulfide $\left(2 \mathrm{H}-\mathrm{MoS}_{2}\right)$ Nano-and Microribbons by Electrochemical/Chemical Synthesis. Nano Lett. 2004, 4, 277-281.

(6) Ross, S.; Sussman, A. Surface Oxidation of Molybdenum Disulfide. J. Phys. Chem. 1955, 59, 889-892.

(7) Tenne, R.; Margulis, L.; Genut, M.; Hodes, G. Polyhedral and Cylindrical Structures of Tungsten Disulphide. Nature 1992, 360, 444-446.

(8) Sano, N.; Wang, H.; Chhowalla, M.; Alexandrou, I.; Amaratunga, G. A.; Naito, M.; Kanki, T. Fabrication of Inorganic Molybdenum Disulfide Fullerenes by Arc in Water. Chem. Phys. Lett. 2003, 368, 331-337.

(9) Redlich, M.; Katz, A.; Rapoport, L.; Wagner, H.; Feldman, Y.; Tenne, R. Improved Orthodontic Stainless Steel Wires Coated with Inorganic Fullerene-Like Nanoparticles of $\mathrm{WS}_{2}$ Impregnated in Electroless Nickel-Phosphorous Film. Dent. Mater. 2008, 24, 16401646.

(10) Alexandrou, I.; Sano, N.; Burrows, A.; Meyer, R.; Wang, H.; Kirkland, A.; Kiely, C.; Amaratunga, G. Structural Investigation of $\mathrm{MoS}_{2}$ Core-Shell Nanoparticles Formed by an Arc Discharge in Water. Nanotechnology 2003, 14, 913.

(11) Ho, W.; Yu, J. C.; Lin, J.; Yu, J.; Li, P. Preparation and Photocatalytic Behavior of $\mathrm{MoS}_{2}$ and $\mathrm{WS}_{2}$ Nanocluster Sensitized $\mathrm{TiO}_{2}$. Langmuir 2004, 20, 5865-5869.

(12) Alkis, S.; Oztas, T.; Aygün, L.; Bozkurt, F.; Okyay, A.; Ortaç, B. Thin Film $\mathrm{MoS}_{2}$ Nanocrystal Based Ultraviolet Photodetector. Opt. Express 2012, 20, 21815-21820.

(13) Wu, H.; Yang, R.; Song, B.; Han, Q.; Li, J.; Zhang, Y.; Fang, Y.; Tenne, R.; Wang, C. Biocompatible Inorganic Fullerene-Like Molybdenum Disulfide Nanoparticles Produced by Pulsed Laser Ablation in Water. ACS Nano 2011, 5, 1276-1281.

(14) Nath, M.; Rao, C. Nanotubes of Group 4 Metal Disulfides. Angew. Chem., Int. Ed. 2002, 114, 3601-3604.

(15) Wu, C.-C.; Jariwala, D.; Sangwan, V. K.; Marks, T. J.; Hersam, M. C.; Lauhon, L. J. Elucidating the Photoresponse of Ultrathin $\mathrm{MoS}_{2}$ Field-Effect Transistors by Scanning Photocurrent Microscopy. J. Phys. Chem. Lett. 2013, 4, 2508-2513.
(16) Radisavljevic, B.; Radenovic, A.; Brivio, J.; Giacometti, V.; Kis, A. Single-Layer $\mathrm{MoS}_{2}$ Transistors. Nat. Nanotechnol. 2011, 6, 147-150.

(17) Shanmugam, M.; Bansal, T.; Durcan, C. A.; Yu, B. Molybdenum Disulphide/Titanium Dioxide Nanocomposite-Poly 3-Hexylthiophene Bulk Heterojunction Solar Cell. Appl. Phys. Lett. 2012, 100, 153901153901-4.

(18) Gourmelon, E.; Lignier, O.; Hadouda, H.; Couturier, G.; Bernede, J.; Tedd, J.; Pouzet, J.; Salardenne, J. $\mathrm{MS}_{2}(\mathrm{M}=\mathrm{W}, \mathrm{Mo})$ Photosensitive Thin Films for Solar Cells. Sol. Energy Mater. Sol. C 1997, 46, 115-121.

(19) Ataca, C.; Ciraci, S. Functionalization of Single-Layer $\mathrm{MoS}_{2}$ Honeycomb Structures. J. Phys. Chem. C 2011, 115, 13303-13311.

(20) Ataca, C.; Sahin, H.; Ciraci, S. Stable, Single-Layer $\mathrm{MX}_{2}$ Transition-Metal Oxides and Dichalcogenides in a Honeycomb-Like Structure. J. Phys. Chem. C 2012, 116, 8983-8999.

(21) Li, T.; Galli, G. Electronic Properties of $\mathrm{MoS}_{2}$ Nanoparticles. J. Phys. Chem. C 2007, 111, 16192-16196.

(22) Li, Y.; Zhou, Z.; Zhang, S.; Chen, Z. $\mathrm{MoS}_{2}$ Nanoribbons: High Stability and Unusual Electronic and Magnetic Properties. J. Am. Chem. Soc. 2008, 130, 16739-16744.

(23) Lebegue, S.; Eriksson, O. Electronic Structure of TwoDimensional Crystals from ab Initio Theory. Phys. Rev. B 2009, 79, 115409.

(24) Huang, Y.; Wu, J.; Xu, X.; Ho, Y.; Ni, G.; Zou, Q.; Koon, G. K. W.; Zhao, W.; Neto, A. C.; Eda, G. An Innovative Way of Etching $\mathrm{MoS}_{2}$ : Characterization and Mechanistic Investigation. Nano Res. 2013, 1-8.

(25) Liu, K.-K.; Zhang, W.; Lee, Y.-H.; Lin, Y.-C.; Chang, M.-T.; Su, C.-Y.; Chang, C.-S.; Li, H.; Shi, Y.; Zhang, H. Growth of Large-Area and Highly Crystalline $\mathrm{MoS}_{2}$ Thin Layers on Insulating Substrates. Nano Lett. 2012, 12, 1538-1544.

(26) Coleman, J. N.; Lotya, M.; O’Neill, A.; Bergin, S. D.; King, P. J.; Khan, U.; Young, K.; Gaucher, A.; De, S.; Smith, R. J. TwoDimensional Nanosheets Produced by Liquid Exfoliation of Layered Materials. Science 2011, 331, 568-571.

(27) Zhan, Y.; Liu, Z.; Najmaei, S.; Ajayan, P. M.; Lou, J. Large-Area Vapor-Phase Growth and Characterization of $\mathrm{MoS}_{2}$ Atomic Layers on a $\mathrm{SiO}_{2}$ Substrate. Small 2012, 8, 966-971.

(28) Zelenski, C. M.; Dorhout, P. K. Template Synthesis of nearMonodisperse1Microscale Nanofibers and Nanotubules of $\mathrm{MoS}_{2}$. J. Am. Chem. Soc. 1998, 120, 734-742.

(29) Hsu, W. K.; Chang, B. H.; Zhu, Y. Q.; Han, W. Q.; Terrones, H.; Terrones, M.; Grobert, N.; Cheetham, A. K.; Kroto, H. W.; Walton, D. R. An Alternative Route to Molybdenum Disulfide Nanotubes. J. Am. Chem. Soc. 2000, 122, 10155-10158.

(30) Compagnini, G.; Sinatra, M. G.; Messina, G. C.; Patanè, G.; Scalese, S.; Puglisi, O. Monitoring the Formation of Inorganic Fullerene-Like $\mathrm{MoS}_{2}$ Nanostructures by Laser Ablation in Liquid Environments. Appl. Surf. Sci. 2012, 258, 5672-5676.

(31) Amendola, V.; Meneghetti, M. Laser Ablation Synthesis in Solution and Size Manipulation of Noble Metal Nanoparticles. Phys. Chem. Chem. Phys. 2009, 11, 3805-3821.

(32) Liang, C.; Shimizu, Y.; Sasaki, T.; Koshizaki, N. Preparation of Ultrafine $\mathrm{TiO}_{2}$ Nanocrystals via Pulsed-Laser Ablation of Titanium Metal in Surfactant Solution. Appl. Phys. A: Mater. Sci. Process. 2005, $80,819-822$.

(33) Hohenberg, P.; Kohn, W. Inhomogeneous Electron Gas. Phys. Rev. 1964, 136, B864.

(34) Kohn, W.; Sham, L. J. Self-Consistent Equations Including Exchange and Correlation Effects. Phys. Rev. 1965, 140, A1133.

(35) Kresse, G.; Furthmüller, J. Efficient Iterative Schemes for ab Initio Total-Energy Calculations Using a Plane-Wave Basis Set. Phys. Rev. B 1996, 54, 11169.

(36) Kresse, G.; Hafner, J. Ab Initio Molecular Dynamics for Liquid Metals. Phys. Rev. B 1993, 47, 558.

(37) Perdew, J. P.; Burke, K.; Ernzerhof, M. Generalized Gradient Approximation Made Simple. Phys. Rev. Lett. 1996, 77, 3865. 
(38) Grimme, S. Semiempirical Gga-Type Density Functional Constructed with a Long-Range Dispersion Correction. J. Comput. Chem. 2006, 27, 1787-1799.

(39) Blöchl, P. E. Projector Augmented-Wave Method. Phys. Rev. B 1994, 50, 17953.

(40) Tenne, R. Inorganic Nanotubes and Fullerene-Like Nanoparticles. Nat. Nanotechnol. 2006, 1, 103-111.

(41) Seo, J. w.; Jun, Y. w.; Park, S. w.; Nah, H.; Moon, T.; Park, B.; Kim, J. G.; Kim, Y. J.; Cheon, J. Two-Dimensional Nanosheet Crystals. Angew. Chem., Int. Ed. 2007, 46, 8828-8831.

(42) Sharifi, T.; Gracia-Espino, E.; Barzegar, H. R.; Jia, X.; Nitze, F.; Hu, G.; Nordblad, P.; Tai, C.-W.; Wågberg, T. Formation of NitrogenDoped Graphene Nanoscrolls by Adsorption of Magnetic $\Gamma-\mathrm{Fe}_{2} \mathrm{O}_{3}$ Nanoparticles. Nat. Commun. 2013, 4.

(43) Enyashin, A. N.; Bar-Sadan, M.; Sloan, J.; Houben, L.; Seifert, G. Nanoseashells and Nanooctahedra of $\mathrm{MoS}_{2}$ : Routes to Inorganic Fullerenes. Chem. Mater. 2009, 21, 5627-5636.

(44) Chuvilin, A.; Kaiser, U.; Bichoutskaia, E.; Besley, N. A.; Khlobystov, A. N. Direct Transformation of Graphene to Fullerene. Nat. Chem. 2010, 2, 450-453.

(45) Perkins, F.; Friedman, A.; Cobas, E.; Campbell, P.; Jernigan, G.; Jonker, B. Chemical Vapor Sensing with Monolayer $\mathrm{MoS}_{2}$. Nano Lett. 2013, 13, 668-673.

(46) Yue, Q.; Shao, Z.; Chang, S.; Li, J. Adsorption of Gas Molecules on Monolayer $\mathrm{MoS}_{2}$ and Effect of Applied Electric Field. Nanoscale Res. Lett. 2013, 8, 1-7.

(47) Ataca, C.; Ciraci, S. Dissociation of $\mathrm{H}_{2} \mathrm{O}$ at the Vacancies of Single-Layer $\mathrm{MoS}_{2}$. Phys. Rev. B 2012, 85, 195410. 